\title{
The Power Supply Module Design for Diesel Electronic Control Unit's Lift Time Test System
}

\author{
Jinguan Yin ${ }^{1}$, Tiexiong Su${ }^{1}$, Xiaoyan Shen ${ }^{2}, \mathrm{Li} \mathrm{Jia}{ }^{2}, \mathrm{Tao} \mathrm{Liu}^{2}$ and Hui Lv² \\ ${ }^{1}$ College of Mechatronic Engineering, North University of China, Taiyuan, Shanxi 030051, China \\ ${ }^{2}$ China North Engine Research Institute, Tianjin, 300400, China
}

\begin{abstract}
In this article we focus on the voltage signals supply to power-on-off testing and some specific fault cases for diesel engine electronic control unit life time test system. According to the actual engineering needs we design six different voltage signals supply modules and use TESTBASE bench to supply power to diesel electronic control unit directly. These voltage signals supply modules are all used to supply varied power-on-off environment so we can observe the status of electronic control unit visualized. The accuracy and reliability of voltage power supply module are verified by simulation and experiment. The result shows it provides a good environment for diesel electronic control unit life time test.
\end{abstract}

\section{Introduction}

Almost all the diesel engine use electronic control nowadays. Facing the fault condition such as lightning, static shock, load dump et.al, the diesel engine electronic control unit (ECU) power supply circuit should protect circuit itself is not damaged by the excessive voltage surge, and guarantee the circuit running right at the same time. In order to ensure the reliability of ECU power supply system, on the one hand we designed related circuit to protect the power supply system; On the other hand we should carry on the relevant test to evaluate of the reliability and stability. Life time test system (LTT) is a real-time simulation system for the ECU product. And nowadays we use hardware-in-loop (HIL) to realize the LTT $[1,2]$, which can has test under different conditions especially the fault and limiting condition [3]. The voltage mutation that causes the single chip of supplementary equipment in and out of the external interrupt frequently, causes stack overflow, the network into a dormant state and couldn't be awakened, easy crash failures in the process of ECU power-on-off. The ECU system should be tested to ensure the reliability and stability of the ECU before it is out of factory. The test process is test system analysis, which is test the influence of wave source to test system using statistics method, giving the final report about the system whether pass muster or not. In engineering, using the repeatability and reproducibility of test system to analyse features usually. And when the ECU system is fault, we should have a fault recurrence to find what fault has happened on the ECU. For the power system, the fault is produced by voltage surge and electromagnetic interference. In order to test the state of ECU in the process of power-on-off and fault recurrence, we design power supply module contains six different voltage signals models satisfy LTT requirements.

\section{Test environment}

As shown in Fig. 1, Simulink model, VeriStand software as a PC interface, TestBase bench and real ECUs constitute the LTT environment.

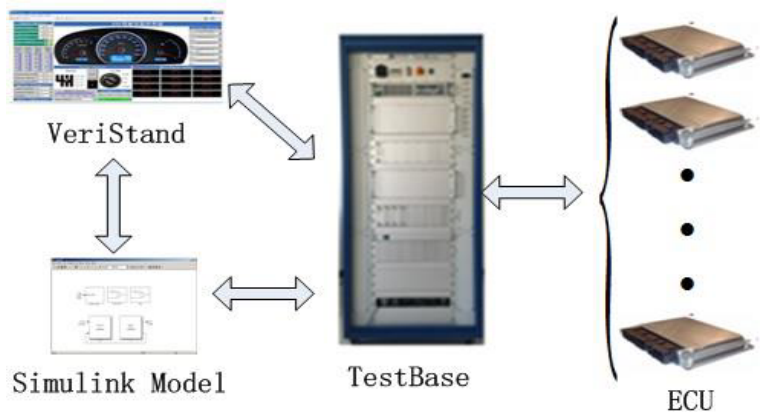

Figure 1. The environment of life time test system

A series of test and analysis hardware equipment comprise the TestBase bench as shown in Table 1. TestBase bench connects to VeriStand software using ethernet network.

TestBase bench use EA programmable power supply module which is used to simulate the ECUs power supply system. The voltage and current can be manual control, also can be controlled through real-time computer output port [4]. It can satisfy the ECU power test demand, such as voltage up and down surge, voltage over high and low limits, wave voltage etc. operating mode, especially the 
vehicle power is in short supply and voltage surge to power supply system when engine start. Fig. 2 (a) is a picture of EA programmable power supply module, Fig. 2 (b) is a picture of TestBase bench power supply management unit, and its main performance index as shown is Table 2 .

Table 1. TestBase hardware component

\begin{tabular}{|c|c|}
\hline Hardware & Application \\
\hline Real-time component & Processor, I/O board \\
\hline Signal management unit & Control board signal \\
\hline Load simulation unit & Simulate real load \\
\hline Fault injection unit & Electrical fault injection \\
\hline Vehicle power simulator & $\begin{array}{c}\text { Simulate vehicle power } \\
\text { supply system }\end{array}$ \\
\hline Power supply management unit & $\begin{array}{c}\text { Manage bench power } \\
\text { supply system }\end{array}$ \\
\hline
\end{tabular}

Table 2. Performance index of EA power

\begin{tabular}{|c|c|}
\hline Version & PS8080-60 \\
\hline Voltage range & $0-60 \mathrm{~V}$ \\
\hline Current range & $0-80 \mathrm{~A}$ \\
\hline
\end{tabular}

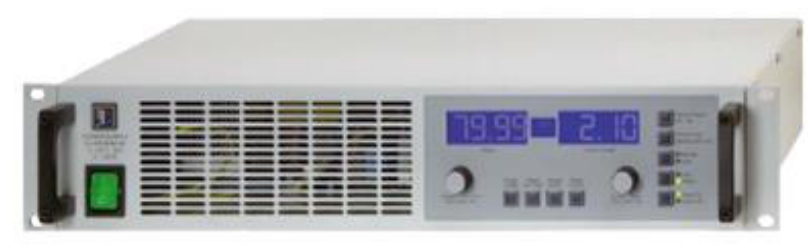

(a)

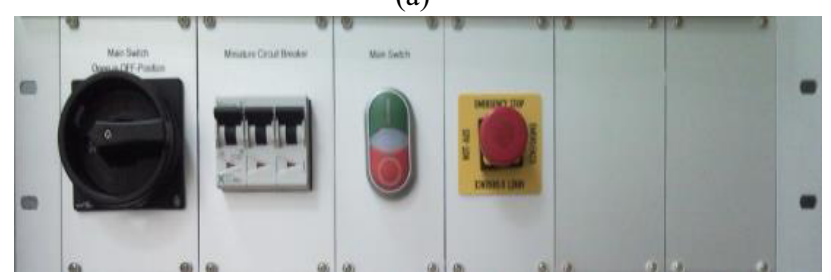

(b)

Figure 2. EA programmable power supply module and power supply management unit

The Simulink model includes the ECU control model, engine model, the $\mathrm{I} / \mathrm{O}$ model and power control model. The engine model simulates the diesel engine, transmitting and receiving the signals from analogue input/output board and digital input/output board through $\mathrm{I} / \mathrm{O}$ model. In the power control model we give our voltage signals arithmetic. In Simulink model, we can design arithmetic expediently and discretionarily.

VeriStand [5] software as a PC interface in the realtime simulation. We designed the power supply module in the Simulink software firstly, and download the arithmetic to TestBase bench. Then we control and observe test process on the VeriStand project. In the VeriStand project, the parameters of the voltage signals arithmetic can be changed easily. It can test more than a dozen ECUs at the same time.

\section{Power control module design}

We designed six modes to supply power for ECU poweron-off in LTT and fault recurrence, it can be arbitrary switched in the testing process. These power control modules are useful in the diesel ECU test, including the hardware and software. The six control mode are DC mode, high-low mode, fixed slope mode, random slope mode, cycle mode and normal mode. These different kinds of power control mode will be described in detail. The overview of the power control module in Simulink can be found in Fig. 3.

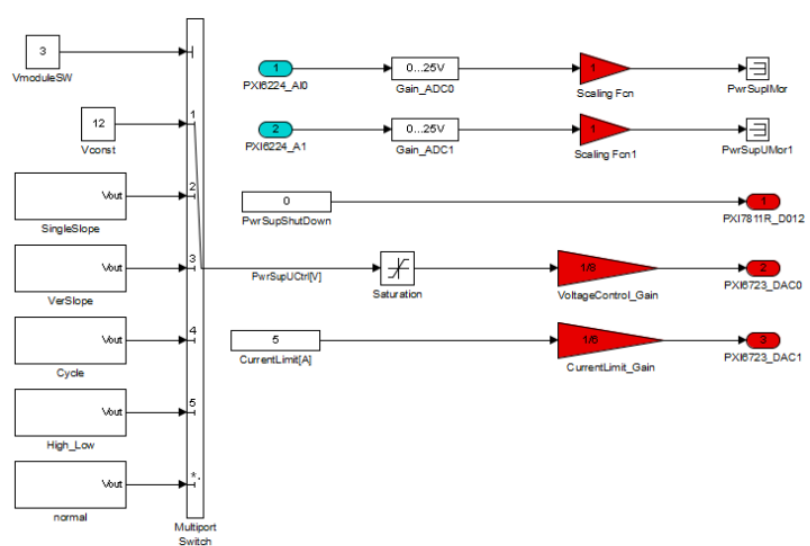

Figure 3. The overview of the power control module

\subsection{DC mode}

The most basic mode is DC power supply, which is giving a constant voltage to ECU. The main function of this mode is to simulate the operating voltage of the ECU in normal work.

$$
V_{k}=C
$$

As shown in Fig. 4, we appoint $C$ is $12 \mathrm{~V}$, in the DC mode the experimental voltage output has an error with the ideal voltage output, but the error is negligible. The voltage can meet the demand of normal working. In practice, the electric wire between TestBase and ECUs should be as short as possible, since the electric wire has a voltage drop effect.

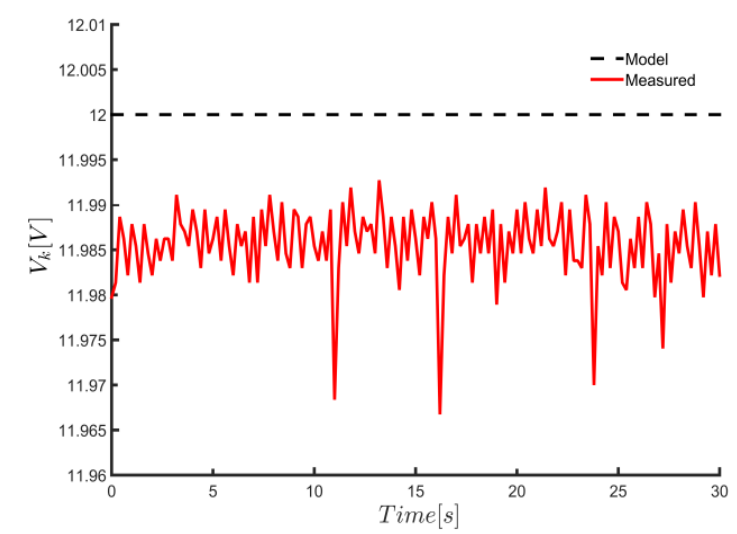

Figure 4. Voltage output to ECU in DC mode 


\subsection{High-Low mode}

For simulating the ECU sudden power-on-off and some other special circumstances, we use high and low level voltage signals to ECU.

$$
\begin{aligned}
& V_{k}=\left\{\begin{array}{l}
V_{\text {High }}, t=t_{\text {High }} \\
V_{\text {Low }}, t=t_{\text {Low }}
\end{array}\right. \\
& t_{\text {period }}=t_{\text {High }}+t_{\text {Low }}
\end{aligned}
$$

$\mathrm{V}_{\mathrm{k}}$ is the current voltage output, $\mathrm{V}_{\mathrm{High}}$ is the high level voltage and it's duration is $t_{\mathrm{High}}, \mathrm{V}_{\text {Low }}$ is the low level voltage and it's duration is $t_{\text {Low }}$. All the parameters of voltage and time are variable in the process of test. The simulation and experiment result is shown in Fig. 5. This mode is used for testing how the ECU power sudden up and down influences the ECU hardware and software. If the software isn't designed well, it may causes stack overflow, the network into a dormant state and couldn't be awakened.

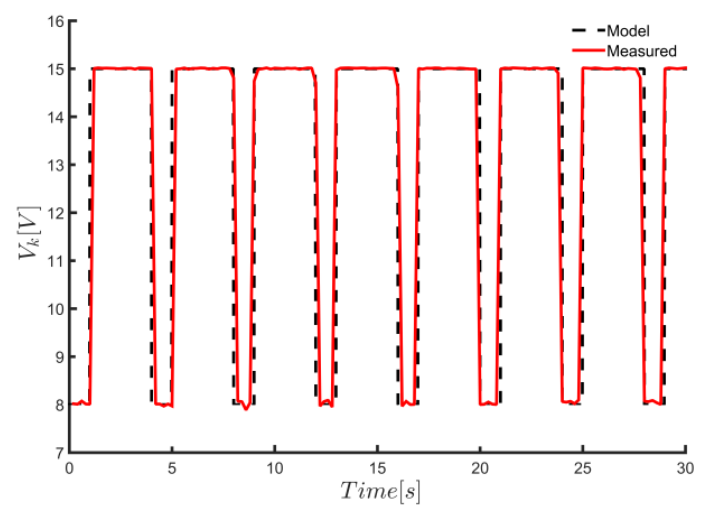

Figure 5. Voltage output to ECU in high-low mode

In this case, we appoint $V_{\text {High }}$ is $15 \mathrm{~V}, V_{\text {Low }}$ is $8 \mathrm{~V}$, $t_{\text {High }}$ is $3 \mathrm{~s}$ and $t_{\text {Low }}$ is $1 \mathrm{~s}$. As shown in Fig. 3 when the high-low level changed, the voltage is not changed immediately in experiment. But the voltage is changed very fast, so it can reach the design demand.

\subsection{Fixed slope mode}

In this mode, we set the minimum voltage and the maximum voltage. The TESTBASE's output voltage reciprocate between the maximum and minimum voltage, and have a fixed increase/decrease slope. Using a state machine controls the output voltage, when monitoring the last moment output voltage is less than the maximum voltage, then output voltage will be the sum of the last moment voltage and the rising slope until the output voltage over the maximum voltage. When the output voltage reached the maximum voltage, the output voltage will be the sum of the voltage and the down slope until the output voltage less than the minimum voltage. The next cycle will occur after a waiting time.

$$
V_{k}=\left\{\begin{array}{c}
V_{k-1}+\text { UpSlope }_{\text {fixed }}, V_{k-1} \leq V_{\text {min }} \\
V_{k-1}+\text { DownSlope }_{\text {fixed }}, V_{k-1} \geq V_{\text {max }}
\end{array}\right.
$$

where $V_{k}$ is the current voltage output, $V_{k-1}$ is the last moment voltage output, UpSlope fixed is the fixed increase slope, DownSlope fixed $_{\text {is }}$ the fixed decrease slope. Similarly we can appoint the values of these parameters on PC interface in the test. The simulation and experiment results as shown in Fig. 6.

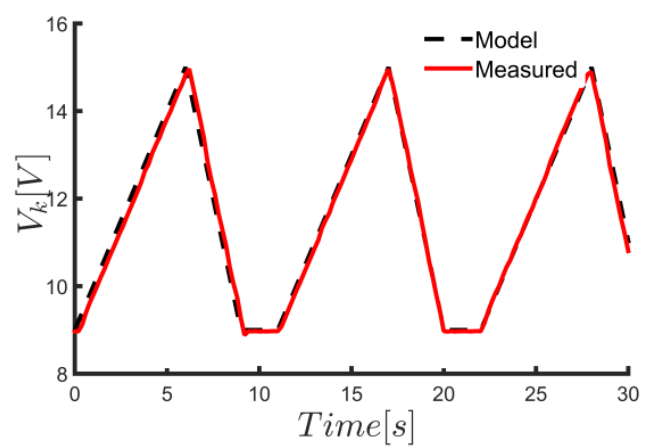

Figure 6. Voltage output to ECU in fixed slope mode

In this case we appoint $V_{\text {High }}$ is $15 \mathrm{~V}, V_{\text {Low }}$ is $9 \mathrm{~V}$, UpSlope $_{\text {fixed }}$ is increased $2 \mathrm{~V}$ per minutes, DownSlope $_{\text {fixed }}$ is decreased $2 \mathrm{~V}$ per minutes and the waiting time is $2 \mathrm{~s}$. Observing the status of ECU when the voltage up and down slowly. This mode is used for ECU power-on-off sluggish and the fault recurrence.

\subsection{Random slope mode}

Unlike the fixed slope mode, the random slope mode has a random increase/decrease slope. The step of model is $0.001 \mathrm{~s}$, so we give a random value as the slope in every step. Voltage is changed faster and the waiting time is shorter than other modes in random slope mode, this is in order to test the performance of the relay and the performance of ECU hardware and software. The formula of random slope mode is similar with fixed slope mode formula as:

$$
V_{k}=\left\{\begin{array}{c}
V_{k-1}+\text { UpSlope }_{\text {random }}, V_{k-1} \leq V_{\text {min }} \\
V_{k-1}+\text { DownSlope }_{\text {random }}, V_{k-1} \geq V_{\text {max }}
\end{array}\right.
$$

where $\mathrm{V}_{\mathrm{k}}$ is the current voltage output, $\mathrm{V}_{\mathrm{k}-1}$ is the last moment voltage output, UpSlope random $_{\text {is }}$ the random increase slope, DownSlope random $_{\text {is }}$ the random decrease slope. The simulation and experiment results as shown in Fig. 7.

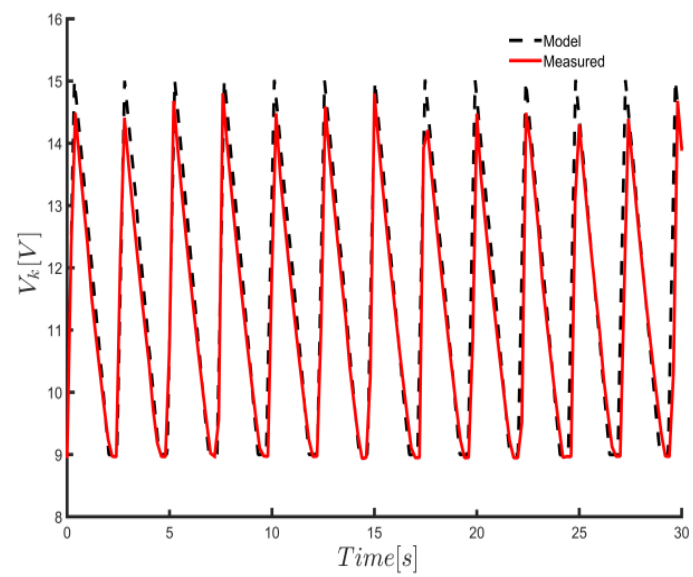

Figure 7. Voltage output to ECU in random slope mode 
In the test we appoint $V_{\text {High }}$ is $15 \mathrm{~V}, V_{\text {Low }}$ is $9 \mathrm{~V}$, but in Fig. 7 we can see it cannot reach the highest voltage in experiment. This is because in most case the random number is too large results the calculative voltage is beyond the upper limit. The state machine determines the result is greater than the highest voltage and transfers the state to decrease.

\subsection{Cycle mode}

In some special occasions, we supply a cycle voltage wave to ECU. We also set the minimum voltage and the maximum voltage in a cycle, and numerable fixed slope mode have different fixed increase/decrease slopes compose one cycle voltage wave. Using this mode to test the reliability of ECU and to observe whether the ECU will fail in some special environment. And all the tests have good repeatability and automation in the HIL test[6,7].

$$
V_{k, i}=\left\{\begin{array}{c}
V_{k-1, i}+\text { UpSlope }_{i}, V_{k-1} \leq V_{\text {min }} \\
V_{k-1, i}+\text { DownSlope }_{i}, V_{k-1} \geq V_{\text {max }} \\
(i=2,3 \ldots)
\end{array}\right.
$$

In practice, we find that in one voltage wave cycle set up three or four different slopes generally can meet the requirement of testing work. The simulation and experiment results are shown in Fig. 8.

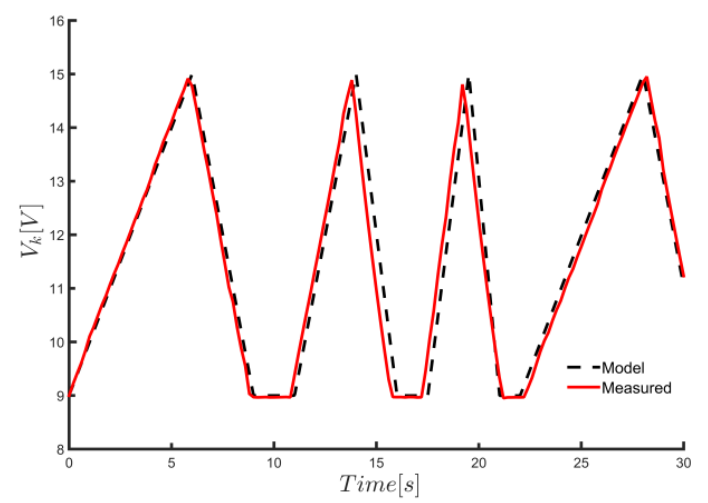

Figure 8. Voltage output to ECU in Cycle mode

In this article we set three different slopes, and $\mathrm{V}_{\text {High }}$ is $15 \mathrm{~V}, \mathrm{~V}_{\text {Low }}$ is $9 \mathrm{~V}$. The first fixed slope mode's increase/decrease slope is $1 / 2$, the second is $2 / 3$ and the third is $3 / 4$. The waiting time is $1.5 \mathrm{~s}$.

\subsection{Normal start mode}

In diesel engine normal start process, there has a surge voltage since the starter working or increasing load over the range of working voltage suddenly. The single chip of supplementary equipment would be in and out of the external interrupt frequently, causes stack overflow, the network into a dormant state and couldn't be awakened, easy crash failures in the process of ECU power-on-off since the voltage mutation. These faults lead to ECU product quality decline. So the test about ECU power in normal start status should be carried though.

In normal start mode, the voltage will decrease to $6 \mathrm{~V}$ and back to normal voltage in a short time. Using this mode to test the reliability of ECU circuit and to observe whether the ECU will fail in the start condition. The simulation and experiment results are shown in Fig. 9. For the purpose of simulate real diesel engine start process, we give a white noise to the voltage since the voltage from TestBase bench and simulation result is tight agreement.

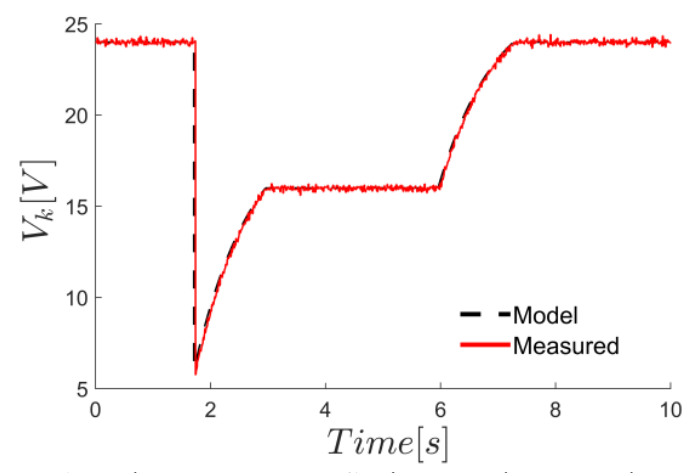

Figure 9. Voltage output to ECU in normal start mode

\section{Conclusion}

According to the engineering needs we designed six kinds of power supply mode for ECU life time test and fault recurrence. DC mode supplies constant voltage for ECU normal work. High-Low mode make ECU poweron-off conventional, since the high and low level voltage transform instantaneously and the high level is hold on for a period of time. Fixed slope mode and Cycle mode have a andante slope, we designed these for make ECU power-on-off sluggish and the fault recurrence. Random slope mode is used for testing the performance of the relay and ECU hardware and software, and this mode has a fast up/down slope. The normal start model simulates the real engine start process, it can test ECUs using surge voltage. The modes and parameters in each mode can be changed manually on PC interface, or they can be written in automated test cases. The above six kinds of modes can meet the requirements of the life test for power supply basically, and we can add more mode if we have other requirement.

\section{References}

1. K. Lamberg, M. Beine, M. Eschmann, etc. SAE Paper, 01-1593 (2004)

2. C. Washington, J. Dolman. IEEE Autotestcon, 1-6 (2010)

3. W. Bi, JM. Wang. 3rd International Conference on Advanced Computer Theory and Engineering, 177181 (2010)

4. CP. Young, WL. Juang, MJ. Devaney. IEEE T INSTRUM MEAS, 49, (3), 579-584 (2000)

5. Nation Instruments White Paper, https://decibel.ni.com/content/docs/DOC-5684

6. A. Palladino, G. Fiengo, F. Giovagnini. IEEE European Control Conferenc, (2009)

7. Z. Liu, N.Gu. Proceedings of 2005 The Fifth International Conference on Computer and Information Technology, 1-3 (2005) 